\title{
La reforma psiquiátrica hoy día en Trieste e Italia ${ }^{1}$.
}

The psychiatric reform today in Trieste and Italy.

Pasquale Evaristo.

Psiquiatra. Departamento Salud Mental de Trieste.

pasqualino.evaristo@gmail.com

La locura está dentro de la normalidad; esta es una de las bases de la revolución psiquiátrica de Basaglia y de su grupo de Trieste.

Las ideas y el trabajo de Franco Basaglia, psiquiatra de Venecia, director del hospital psiquiátrico de Gorizia desde 1961 (intentó humanizarlo) y después director del hospital psiquiátrico de Trieste (1971-79) (completó el camino cerrándolo) lo convirtieron en el verdadero padre de la psiquiatría sin manicomios.

Hizo su formación en la antigua Universidad de Padua y tuvo contactos estrechos con la Fenomenología alemana y con todos los movimientos de crítica institucional, filosófica y social sobre la psiquiatría y la diversidad, que nacieron en el mundo occidental después la $2^{\circ}$ guerra mundial: con Francia (psiquiatra de sector de Daumezon y Bonafè, psicoanálisis institucional, filósofos y sociólogos como Sartre, Foucault, Castel, Guattari..), con Inglaterra (antipsiquiatría de Laing y Cooper, comunidad terapéutica de Maxwell Jones..), con USA ( movimientos para los derechos humanos, Saz, Schatzman, la reforma de la Mental Health Act de Kennedy...). La teoría de Basaglia parece ya bien conocida en España y solo haré algunas alusiones.

Basaglia inventó la desinstitucionalización de las instituciones, fundamento de la experiencia de la reforma de Trieste (junto a la Fenomenología con su atención a las personas) que consiste en reformar lo interior, trabajando desde dentro de las instituciones que con el tiempo tienden a cerrarse en sus reglas creando situaciones desproporcionadas y corruptas, para modificarlas o anularlas, defendiendo así los derechos de los ciudadanos. Esto precisa un trabajo interno y no solo una reforma hecha de lejos, por que el contacto día a día va creando conciencia de los dispositivos institucionales, evitando inercias y generando cambios.

La estrategia de la desinstitucionalización prevé varios niveles: 1. culturalcientífico; 2. organizativo; 3. relaciones humanas. Esta estrategia es la verdadera especificidad técnica en salud mental, no tanto los diferentes enfoques psicoterapéuticos.

Hoy como ayer todas las instituciones en las que vivimos, la familia, la escuela, nuestro trabajo en salud mental...son vividas probablemente como un marco positivo. Pero esto no es automático. Intentamos vivir con actitud crítica, anti-

\footnotetext{
${ }^{1}$ Conferencia invitada a XXVII Jornadas de la Asociación Andaluza de Neuropsiquiatría (AEN). Córdoba, 4-6 noviembre 2010.
} 
institucional nuestro abordaje en salud mental. Basaglia inventó así posibilidades continuas de cambio.

La atención a los internados fue contemporánea a las intervenciones -institucionales y esta estrategia se mantiene actualmente. Antes, con personas aniquiladas por la institución manicomial durante tantos años, se precisaba reconstruir contactos humanos, estimulando confianza comunicativa, y sobre todo reconstruyendo su identidad. Ahora pacientes alienados por mecanismos sociales y familiares precisan de la misma atención personal pero también de atención a su contexto, interactuando y englobando las diferentes instituciones.

La ley 180, generada por el trabajo antes mencionado, quizá la única ley nacional que prohíbe totalmente hospitales psiquiátricos, es un marco general de pocos y claros elementos:

- el cierre gradual de los hospitales psiquiátricos, prohibiendo nuevos ingresos y la construcción de nuevos hospitales; el cierre completo precisó de más de 15 años.

- la apertura de Centros de Salud Mental (C.S.M.) Comunitarios, ejes de la nueva red asistencial para prevención, cura y rehabilitación de pacientes psíquicos.

- pequeñas secciones de psiquiatría en los hospitales generales (máximo 15 camas, para que no vuelvan a la atmosfera manicomial), si los pacientes no tienen oportunidad de ingresar en los C.S.M.

- conexiones con los otros sectores de la medicina dentro del Sistema Nacional de Salud ; antes la psiquiatría tenia una Administración separada, bajo normas del Ministerio del Interior.

- los tratamientos deben ser voluntarios; los obligatorios deben ser excepciones: TSO (tratamientos sanitarios obligatorios) precisan de garantías especificas (firmas de dos médicos, por lo menos uno psiquiatra publico, ratificación del alcalde como representante de los ciudadanos, control formal del juez) y por tiempo breve ( 7 días, renovables con las mismas garantías).

- transferir gradualmente los recursos (trabajadores, dinero...) del hospital a los Servicios Comunitarios (C.S.M.).

El Plan de actuación, que tenia que llegar pronto para dar indicaciones concretas, fue establecido solo en 1994 por resistencia y inercia política, que tal vez continúa.

Esta fue una causa de la escasa o diferente aplicación de la ley en los diferentes lugares de Italia (como manchas de una piel de leopardo, sobre todo al comienzo), junto a diferentes sensibilidades de profesionales y administradores locales, en organizar Servicios y preparar pacientes y familias para el regreso a la sociedad.

La Ley 180 fue también una ley revolucionaria por sus nuevos paradigmas científicos, que fastidiaba los barones académicos, despertaba miedos escondidos 
PARA EL DEBATE

hacia los locos libres, combatía privilegios económicos y de comodidad (el mercado paralelo de los psiquiatras públicos y de las clínicas privadas,) obligo a trabajar más y más cerca del paciente). "Si los pacientes están encerrados, el psiquiatra está libre; si los pacientes están libres, el psiquiatra está preso" (F. Basaglia).

El Plan Nacional actual POSM $2^{\circ}$ de 1998-2000 (Plan Objetivo de Salud Mental) da indicaciones muy específicas:

- crear una red única de Servicios públicos de Salud Mental, organizada como Departamento de Salud Mental (DSM), con recursos específicos y dirección única, dividido en unidades operativas como C.S.M. (1 por lo menos cada 150000 hab.).

- organizar en todo el país las diferentes estructuras necesarias para los diferentes niveles de demanda: Servicio hospitalario para agudos, C.S.M. como eje central de programas y acciones terapéutico-rehabilitadoras, centro de día para post crisis, hogares protegidos para discapacitados, convenciones con cooperativas sociales de trabajo para rehabilitación laboral.

- definir los estándar de servicios cada 10000 hab. (1 cama para agudos, 1cama de día para subagudos, 1 cama en hogares protegidos) y de profesionales (1 cada 1500 hab.), parámetros no siempre cumplidos.

- aclarar funciones asistenciales: continuidad terapéutica con actitud activa hacia los pacientes y el malestar juvenil, plan terapéutico individual, integración entre Servicios de S. M. y otros Sanitarios Regionales y Sociales Municipales (que tienen deberes a nivel de subsistencia y vivienda para los ciudadanos vulnerables).

- capacitar profesionales.

- organizar sistemas de evaluación de Servicios, éxitos, satisfacciones de ciudadanos...

Durante varios años, diferentes grupos parlamentarios hicieron propuestas de cambio, también en el extranjero, donde se discutía de reformas psiquiátricas.

Hoy día la ley 180 tiene mas de 30 años de vida y la mayoría de personas implicadas (profesionales, pacientes, familiares, administradores, asociaciones, empresarios laborales del sector privado-social...) y de los partidos políticos no piensan en mudarla, si no en continuar a implementándola. Pero en este ultimo gobierno Berlusconi llegaron otras propuestas de cambio parcial, que expresaban sobre todo miedo a la peligrosidad de los que son diferentes (inmigrantes y ladrones más que locos), pidiendo TSO mas largo y sin tantas garantías. Pero eso parece mas un intento publicitario que una realidad, o en todo caso posible apertura a iniciativas privadas en salud mental.

¿Cuál es la realidad de los Servicios de S. M. comunitaria en Italia?

Todavía está bastante diversificada, pero menos que antes. Ahora en todas las regiones hay un organización en Departamentos de S. M., estructurales o funcionales, que administran el circuito público (camas en Hospitales Generales para 
agudos; Centro de S. M. comunitarios para programas de atención personales, pero con horarios de atención diferentes: La mayoría con 8 o 12 h días laborables, pocos con $24 \mathrm{~h}$ y abiertos en días festivos, otros casi solo ambulatorios; Comunidades terapéuticas a veces contratadas con circuitos privados...).

Tenemos lugares que continúan desarrollando la ley 180, pero bajo el desafío con los límites económicos, y otras regiones, por ejemplo del norte, que actúan medicalizando los Servicios Hospitalarios y comunitarios y privatizando estructuras de rehabilitación. También Trieste esta adentro de este contexto económico y político, y con las próximas elecciones municipales y provinciales de primavera, podría pasar que ninguna institución gubernamental, nacional o local, apoye nuestro trabajo y que pidan reducción de fondos y de proyectos.

Pero hasta ahora Trieste fue el ejemplo a nivel de ideas y también de organización. Tenemos los Servicios indicados en el POSM '98 desde muchos años, con más algunas características:

- dentro una área de 240000 hab. (Provincia toda) un Departamento de S. M. (DSM), dividido en 4 C.S.M. (cada uno con un área de mas o menos 60000 hab. igual que un Distrito de Salud general), donde hay también 6-8 camas para agudos, cuidados, consultas e intervenciones de grupo con pacientes, familiares y mucho trabajo domiciliario e interconsultas en hospital general, residencias de mayores, cárcel..Conexiones continuas con Salud Publica del área sobre problemas comunes (atención $1^{\circ}$, consultorio familiar, menores, Alzheimer, minusvalías, toxicómanos...).

- 1 Servicio hospitalario con 6 camas para observación; la hospitalización normalmente se hace en el C.S.M.

- 1 Servicio de Habilitación (sobre todo inserción laboral) y Hogares protegidos.

- enlace con Cooperativas Sociales de trabajo (han de tener al menos un 30\% de socios con minusvalías), Asociaciones de familiares y voluntarios, Club de autoayuda de pacientes.

- parte del presupuesto, o sea mas de 435.000 euros/año, dedicado a actividades extra clínicas (inserciones laborales, intervenciones de socialización, de tiempo libre y deportes, subvenciones para necesidades...), necesarias para programas individualizados postcrisis.

¿Qué éxitos tenemos en el DSM de Trieste?

- de 1300/ano internados forzosos en hospital psiquiátrico en 1971 a unos 30 pacientes/año con TSO.

- no hay pacientes internados in Hospitales Psiquiátricos Judiciales (1500 camas en Italia, pero ahora están en un proceso de regionalización).

- 4.500 pacientes/año que acuden voluntariamente con diferentes niveles de malestar: mas de 100 cada día en cada CSM, pocas visitas/día en el Servicio Hospitalario.

- en lugar de una única respuesta al enfermo mental grave (internamiento 
PARA EL DEBATE

forzoso en Hospital psiquiátrico) ahora hay alrededor de 50 lugares diferentes con programas lo mas personalizados posibles.

- no había ni hay clínicas psiquiátricas privadas para internamiento en nuestra región.

- los pacientes conservan todos los derechos de ciudadanía y viven dentro de la comunidad, con apoyos diferentes según las situaciones personales de cada uno en cuanto a su enfermedad o dificultades. hock.

- no se utiliza contención mecánica (pero atención mas intensiva) ni electros-

- han nacido auto-organizaciones de familiares y otras de pacientes, que colaboran con los Servicios.

- los costes del hospital psiquiátrico del año 1971 eran el doble de los de hoy.

- los edificios y el parque del hospital psiquiátrico están casi todos reutilizados por otras instituciones de ciudadanos (escuelas, residencias, espacios culturales...).

- el estigma en la ciudad es menor que antes (investigación '06 con 900 hab. de Trieste).

- centenares de pacientes encontraron trabajo a través la inserción laboral de los C.S.M.

Nuestros indicadores de Servicios de S. M. Comunitarios, que garanticen el tratamiento y el respecto de los derechos de los ciudadanos serian:

1. Verdadera RED UNICA de Servicios Públicos de Salud Mental (DSM) para integrar las diferentes áreas y competencias, e impedir estrategias conflictivas y abandono de pacientes, organizando la responsabilidad política y profesional de la atención sanitaria sobre una área. Eso ha permitido (permite) una reconversión de los recursos hospitalarios a la atención comunitaria y a nuevas necesidades de las personas usuarias.

2. MODELO de referencia no solo medico (según el modelo del Hospital Psiquiátrico y sus terapias), sino BIO-PSICO-SOCIAL para afrontar las diferentes caras del sufrimiento psíquico.

3. C.S.M. comunitarios ACCESIBLES (apertura 24 horas/día, 7 días por semana) con acogida en tiempo real en el día, sin filtros, en conexión con las instituciones sanitarias, sociales y comunitarias del área. La institución se acerca al ciudadano.

4. Asumirse y tratar con CONTINUIDAD los casos mas graves, utilizando también profesionales no-médicos como operadores de referencia (similitud con Modelo Asertivo Comunitario de Avilés, procurando alternativas a la hospitalización).Desarrollar programas rehabilitadores personalizados a nivel domiciliario y comunitario. Escuchar, motivar, hacer juntos el camino.

5. Activar, involucrar y sostener REDES REALES de apoyo entorno al paciente (familiares, amigos, colegas, voluntarios...y otros Servicios no específicos 
de la área) crea a largo plazo un pronostico mejor (Cifras de la OMS '76 sobre investigaciones de éxitos en esquizofrenia). Para construir salud mental es precisa la colaboración entre profesionales y no profesionales.

6. Una ATENCION INTERSECTORIAL con Médicos Generales, Distritos Sanitarios, Unidades Municipales de Apoyo Social, Viviendas Publicas, Asociaciones Locales de Ciudadanos, sobre todo en áreas problemáticas (En Trieste el Proyecto Micro áreas) favorece la prevención de las Enfermedades (Indicadores Sociales de Salud) o su gravedad.

7. Nuestra estrategia de REHABILITACION: Primero rehabilitar las instituciones de la rehabilitación y después a los pacientes, utilizando de manera diferentemente los recursos públicos, orientados a menudo hacia el control más que hacia la emancipación. Crear accesos reales a los derechos de ciudadanía a través recursos concretos en sus lugares de vida y construcción de capacidades de acceso (capacitación y acompañamiento de pacientes). La discapacitación del paciente empieza pronto con el alejamiento de los pacientes agudos de sus lugares, deberes, afectos... es preciso tener una actitud terapéutico-rehabilitadora temprana. Los factores no específicos (historia personal, afectos, actitudes, sentido personal del proyecto, nuestra mirada o expectativa...) prevalecen en la producción del resultado.

8. El TRABAJO constituye el nivel máximo de rehabilitación por que construye/precisa de muchas habilidades (higiene, autonomía, relación...) y produce una gran recuperación de valores sociales si está realizado en lugares reales y no artificiales, con sentido personal, creando productos de calidad y no pasatiempos. Contra el prejuicio de que los esquizofrénicos no pueden trabajar, una investigación del Instituto Nacional M. Negri sobre 2000 pacientes, después 5 anos de tratamiento en Servicios Comunitarios Italianos, demostró que $62 \%$ trabajan.

9. No utilizar la intervención especializada psiquiátrica para hacer solo diagnósticos y etiquetes, que permitan a las otras instituciones alejarse del paciente y verlo absorbido por la enfermedad mental, negando sus derechos de otras intervenciones; es preciso construir espacios de comprensión y ENCUENTRO ENTRE NORMALIDAD Y DIVERSIDAD dentro de todos los lugares donde actuamos (C.S.M., Hospital general, médicos de familia, Comunidad, Familias...). Contra el prejuicio de la peligrosidad, que aleja las personas, también el delito equipara locos y normales (no hay diferencia estadística) y de la misma manera la pena debe ser evaluada igual; solo después entran las circunstancias atenuantes de una enfermedad y las necesidades de tratamiento, en cárcel o en Servicios Sanitarios.

10. No olvidar que la DESINSTITUCIONALIZACION es una lucha continua dentro de todas las instituciones humanas, las hospitalarias y asistenciales, y las comunitarias, los medios de información pero también las de la vida diaria, para abrirlas a las necesidades cambiantes de las personas y evitar que estas, sin acogida, se cronifiquen. Dar voz directa a los ciudadanos pacientes, arriesgar con ellos para el cambio. 
PARA EL DEBATE

Trieste, después de casi 40 años de trabajo en la comunidad, es testigo de que la salud mental se construye no solo con resultados clínicos, si no también a través de una continua lucha en cada conjunto humano, con muchas dificultades para abrir espacios de vida a las personas mas débiles.

Problemas todavía abiertos en Trieste e Italia en el campo psiquiátrico:

- Nadie es perfecto, ni persona ni institución. El trabajo continuo y cercano a personas con trastornos mentales es más difícil y duro que encerrarlas. Se precisa de un trabajo de grupo y de supervisión, para mantener un equilibrio y no caer en el "burnout".

- Los prejuicios sobre la locura son menos, pero quedan. "El manicomialismo", la actitud de poner las contradicciones sociales y las diversidades difíciles en lugares separados y no afrentarlas en su contexto, tiende a reproducirse en nuevos guetos (residencias de mayores, Centro de acogida forzosa para inmigrantes, barrios-gueto de las periferias...). Mudar la cultura de la expulsión es difícil y largo, pero la $1^{\circ}$ etapa del hospital Psiquiátrico fue posible. "Somos minoría; si acaso el proceso de desinstitucionalización se para o vuelve atrás, queda probado que es posible tratar la locura sin manicomios" (F. Basaglia).

- Los derechos de los pacientes psiquiátricos han incrementado (ahora hay grupos de usuarios mas activos), pero no bastante, también por un contexto donde están disminuyendo para todo el mundo (medidas antiterrorismo, ampliación de la distancia entre pobres y ricos en el mundo...). A veces los pacientes, minoría social escondida, están dejados a cargo de sus familiares, sin apoyo publico. Las Administraciones Publicas olvidan y dejan fuera. El mercado del trabajo se abre poco a los vulnerables cuando hay crisis para todo el mundo (R. Warner).

- La cultura de Servicios con respuestas mas complejas, creadas en salud mental, corren el riesgo de mudarse otra vez a respuestas tecnificadas o de control de conductas más que de creación de salud (mercado de las técnicas "psico", necesidad de control estatal sobre nuevos "diferentes"...). Es necesario estimular salud mental favoreciendo redes naturales en los contextos más problemáticos.

- Dificultades de presupuesto en bienestar social y salud publica.

- Insuficiencia de sistemas de evaluación de éxitos de Servicios.

- Mayor capacitación para profesionales todavía demasiado académica y escasa sensibilización continua de los ciudadanos, que van a ser un obstáculo al cambio cultural, mas que otras carencias.

Ahora en Italia y en nuestra región los grupos políticos parecen menos sensibles a valores culturales y sociales, a los derechos de los débiles. Sin barrera política y prevención social "la protección para los vulnerables se convierte en amenaza para los demás" (R. Castel) y dentro de un contexto así, Trieste como símbolo de salud mental para todos, podría caer. 